\title{
Desmame precoce: uma revisão sistemática
}

\author{
Early ablactation: a systematic review
}

Desmame precoz: una revisíon sistemática

Ana Carolina Rocha e Silva ${ }^{1}$, Rafael Pedroso Bastos ${ }^{1 \star}$, Zilma Nazaré de Souza Pimentel ${ }^{1}$.

\begin{abstract}
RESUMO
Objetivo: Investigar os principais fatores que levam ao desmame precoce em crianças menores de 6 meses de idade. Métodos: Trata-se de uma revisão sistemática, com abordagem qualitativa, baseada nas "Diretrizes Metodológicas: Elaboração de revisão sistemática e metanálise de estudos observacionais comparativos sobre fatores de risco e prognóstico" disponibilizada pelo Ministério da Saúde. Foram selecionados 14 artigos principais que seguiam os critérios de elegibilidade estabelecidos. Para compor a discussão foi necessário extrair os dados dos 14 artigos em questão, através de uma ficha de extração de dados Resultados: Dentre os fatores encontrados após análise, obtiveram-se: mãe acreditar que possuí leite fraco ou insuficiente; trauma mamilar; voltar ao trabalho ou estudos; interferências externas sejam elas por conta de algum profissional da área da saúde ou familiar; depressão pós-parto; questões socioeconômicas; etnia e baixo peso da criança. Conclusão: A pesquisa foi de suma importância para a comunidade científica tendo em vista a importância da amamentação da criança para a saúde e bem-estar populacional. Dessa forma, pode-se sugerir a realização de políticas públicas que reduzam o desmame precoce ou rastreamento precoce no prénatal das mulheres que possuem esse risco.
\end{abstract}

Palavras-chave: Aleitamento materno, Desmame precoce, Fatores de risco.

\begin{abstract}
Objective: To investigate the main factors leading to early ablactation in children under 6 months of age. Methods: This is a systematic review, with a qualitative approach, based on the "Methodological Guidelines: Elaboration of systematic review and meta-analysis of comparative observational studies on risk and prognostic factors" made available by the Brazilian of Health. A total of 14 major articles were selected which met the established eligibility criteria. To compose the discussion it was necessary to extract the data of the 14 articles in question, through a datasheet of extraction of data. Results: Among the factors found after analysis, we obtained: mother believing that she has weak or insufficient milk; nipple trauma; return to work or studies; external interference be they on behalf of a health professional or family member; baby blues; socioeconomic issues; ethnicity and low child weight. Conclusion: The research was of paramount importance to the scientific community in view of the importance of breastfeeding the child for health and population well-being. Thus, it may be suggested to carry out public policies that reduce early ablactation or prenatal early screening of women at risk.
\end{abstract}

Key words: Breastfeeding, Early ablactation, Risk factors.

1 Universidade do Estado do Pará (UEPA), Santarém-Pará. *E-mail: rpbastos96@gmail.com. 


\section{RESUMEN}

Objetivo: Investigar los principales factores que llevan al destete precoz en niños menores de 6 meses de edad. Métodos: Se trata de una revisión sistemática, con abordaje cualitativo, basada en las "Directrices Metodológicas: Elaboración de revisión sistemática y metanálisis de estudios observacionales comparativos sobre factores de riesgo y pronóstico" disponibilizada por el Ministerio de Salud. Se seleccionaron 14 artículos principales que seguían los criterios de elegibilidad establecidos. Para componer la discusión fue necesario extraer los datos de los 14 artículos en cuestión, a través de una ficha de extracción de datos. Resultados: Entre los factores encontrados después del análisis, se obtuvieron: madre creer que poseía leche débil o insuficiente; traumatismo mamilar; volver al trabajo o estudios; las interferencias externas sean ellas por cuenta de algún profesional del área de la salud o familiar; depresión postparto; cuestiones socioeconómicas; etnia y bajo peso del niño. Conclusión: La investigación fue de suma importancia para la comunidad científica teniendo en cuenta la importancia de la lactancia materna para la salud y el bienestar de la población. De esta forma, se puede sugerir la realización de políticas públicas que reduzcan el destete precoz o rastreo precoz en el prenatal de las mujeres que poseen ese riesgo.

Palabras clave: Lactancia materna, Desmame precoz, Factores de riesgo

\section{INTRODUÇÃO}

O novo guia de orientação da Organização Mundial da Saúde (OMS) e do Fundo das Nações Unidas para a Infância (UNICEF), "Proteger, promover, apoiar o aleitamento materno em estabelecimentos que prestam serviços de maternidade e recém-nascidos: uma revisão do Hospital amigo da criança”, afirma que a amamentação exclusiva durante os 6 primeiros meses de vida do bebê fornece toda a nutrição e energia essenciais para o crescimento e desenvolvimento neurológico (WHO, 2017).

Além disso, o Ministério da Saúde brasileiro ainda destaca outros pontos referentes as vantagens para a criança como: reduzir a chance de obesidade, alergias, hipertensão, colesterol alto e diabetes, como também, estimula o melhor desenvolvimento da cavidade bucal na criança (BRASIL, 2015). Assim, as taxas de morbidade e mortalidade diminuem em crianças que são amamentadas por mais tempo quando comparadas àquelas que foram desmamadas precocemente (VICTORA CG, et al., 2016).

Ademais, para mulher são muitos os benefícios do aleitamento materno na primeira hora de vida, porque ele ajuda nas contrações do útero reduzindo a hemorragia e ainda fortalece o vínculo de afeto entre mãe e filho (UNICEF BRASIL, 2018). A longo prazo, também se pode citar a redução do risco de desenvolver câncer de mama para a mãe que amamentou seu bebê (OPAS/OMS BRASIL, 2018). Como também, reduzir o risco de uma nova gravidez nos primeiros seis meses, pois a amamentação tem um papel fundamental, de eficácia de até $98 \%$, como anticoncepcional se estiver ocorrendo aleitamento exclusivo e a mulher ainda não tiver menstruado (BRASIL, 2015).

Entretanto, diversos fatores podem afetar o aleitamento materno exclusivo (AME) até os 6 meses de idade do bebê, como também o tempo de duração da prática do amamentar. Nesse contexto, destacam-se fatores como: o núcleo em que as mulheres estão inseridas, as condições de educação, a inserção no mercado de trabalho, e o desempenho dos serviços de saúde. Tendo em vista a importância do aleitamento materno, o desmame deve ser realizado quando a criança estiver em condições adequadas para aceitá-lo, não sendo somente uma opção da mãe (TOMA TS e MONTEIRO CA, 2001).

Infelizmente, ainda que a amamentação seja considerada única, tanto para a saúde da mãe e do bebê, são poucas as mulheres que amamentam no continente americano, mesmo o início sendo quase universal, sua duração como alimentação exclusiva é baixa (OPAS/OMS, 2018). Quando se tem em vista o contexto brasileiro é triste observar que apesar dos esforços nacionais e internacionais para o estímulo do aleitamento, as taxas ainda estão longe do esperado ainda mais quando se tem em vista 0 aleitamento de maneira exclusiva (BRASIL, 2015). 
Haja vista, o desmame precoce tem uma relação direta com a morbimortalidade infantil, assim, esse fato expressa elevada importância na determinação de políticas que auxiliem a prática do aleitamento materno. Pesquisas mostram que esta ação pode atuar como prevenção de seis milhões de mortes de crianças menores de doze meses no mundo (MONTRONE VG e ARANTES Cl, 2000).

Nesse contexto, outra pesquisa em 2010, pelo Instituto de Saúde da Secretaria Estadual de São Paulo (SES/SP), avaliou que a prevalência do AME em menores de seis meses era de $41 \%$ no total das capitais brasileiras. Dentre todas as regiões analisadas, a Região Norte do Brasil obteve um índice de 45,9\% (BRASIL, 2010).

Dessa forma, é de caráter ímpar e de extrema importância a amamentação infantil correta a fim de evitar possíveis complicações. Entretanto, a duração e a forma da amamentação está sendo um problema, pois mesmo que o início dela seja praticamente universal, a continuidade dela não está dentro dos parâmetros desejados. Portanto, é necessário conhecer os fatores que levam a esse desmame precoce para então conseguir preveni-los ou identificá-los de forma a evitar que ele ocorra antes da criança estar preparada para o desmame.

Com essa visão, esse trabalho busca, por meio de uma revisão sistemática, identificar quais são esses fatores que levam ao desmame antes do tempo recomendado, para assim alertar os profissionais da área da saúde e a população em geral a ficarem atentos a respeito dos mesmos com a finalidade de melhorar a saúde materno-infantil.

\section{MÉTODOS}

Trata-se de uma revisão Sistemática de estudos observacionais baseada nas "Diretrizes Metodológicas: Elaboração de revisão sistemática e metanálise de estudos observacionais comparativos sobre fatores de risco e prognóstico" do Ministério da Saúde (BRASIL, 2014).

Para a identificação dos artigos sobre o tema, a pesquisa foi realizada nas bases de dados eletrônicas: LILACS, via BVS; MEDLINE, via PubMed e SciELO. Utilizou-se artigos nos idiomas português, inglês e espanhol com o período de publicação de 2013 a 2017.

Como critério para considerar o estudo, instituiu-se uma questão norteadora da pesquisa: "Quais os fatores que levam ao desmame precoce em crianças menores de 6 meses de idade?"; na qual se estabeleciam o tipo de participante (binômio mãe-filho); tipo de exposição (fatores que levam ao desmame precoce); tipo de controle (desmame em tempo adequado); e tipo de desfecho (desmame precoce).

A coleta e análise de dados foi feita em estudo duplo-cego, em que se foram avaliados de maneira independente os títulos, resumos e artigos completos que preenchiam, os critérios de elegibilidade, através de um Formulário de Elegibilidade, padronizado pelos próprios autores.

Após isso, foi realizada uma reunião, e foram selecionados 14 artigos em destaque (Tabela 1), e que norteavam a presente revisão sistemática.

Em seguida, através de uma Ficha de Extração de Dados, retirava-se as informações dos artigos (população pesquisada e quantidade; estudo metodológico empregado; período de realização do estudo; objetivo do estudo; principais fatores que influenciam no desmame precoce; resultados e conclusão do estudo), que eram gerenciados pelo Formulário de Elegibilidade, que caracterizava os artigos analisando seus tipos de estudos, participantes, exposição e desfecho. O fluxograma (Figura 1) a seguir mostra o processo de triagem. 
Figura 1 - Fluxograma da seleção dos artigos para a revisão sistemática
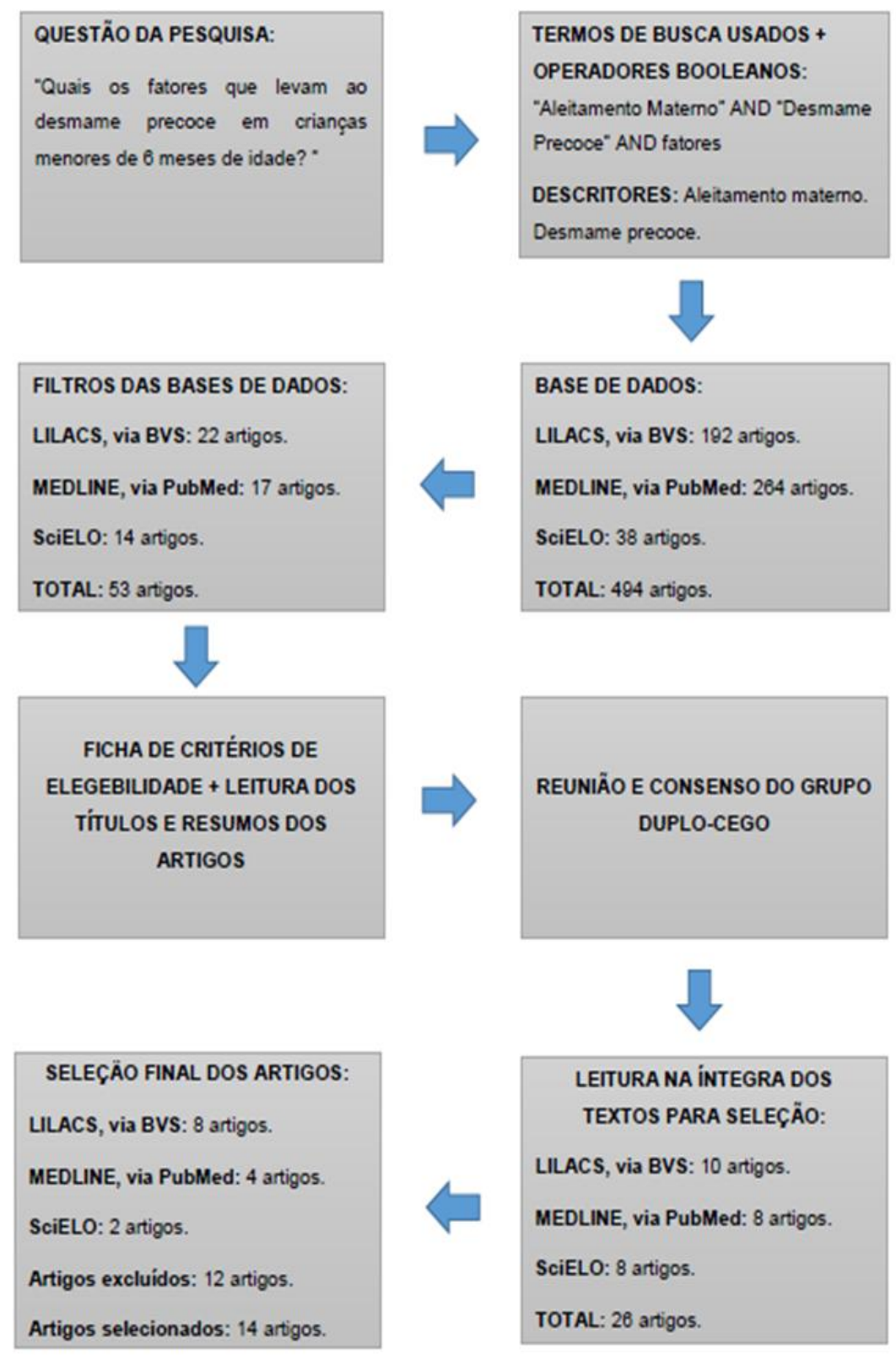

Fonte: Elaborado pelo autor, 2018.

\section{RESULTADOS}

A revisão sistemática do tema proposto encontrou variáveis que tendem a explicar a ocorrência do desmame precoce. Elas são importantes quando se tem em vista o objetivo proposto pela pesquisa.

Após analisar os artigos e escolhê-los de acordo com a metodologia proposta, tem-se a citação dos principais fatores encontrados que levam ao desmame precoce.

Estes são: dúvidas por parte da mãe sejam elas por insegurança, inexperiência ou falta de conhecimento; acreditar que possuí leite fraco ou insuficiente; trauma mamilar; voltar ao trabalho ou estudos; interferências externas sejam elas por conta de algum profissional da área da saúde ou familiar; depressão pós-parto; questões socioeconômicas; etnia; baixo peso da criança e mãe tabagista.

Os artigos selecionados pelos critérios de elegibilidade, com seus respectivos autores, data de publicação, objetivos, tipo de estudo, principais resultados e revista publica estão organizados na Tabela 1. 
Tabela 1 - artigos selecionados pelos critérios de elegibilidade da revisão sistemática.

\begin{tabular}{|c|c|c|c|c|c|}
\hline AUTORIANO & TÍTULO & OBJETIVO & $\begin{array}{l}\text { TIPO DE } \\
\text { ESTUDO }\end{array}$ & $\begin{array}{l}\text { PRINCIPAIS } \\
\text { RESULTADOS }\end{array}$ & $\begin{array}{c}\text { REVISTAI } \\
\text { PUBLICAÇÃO }\end{array}$ \\
\hline $\begin{array}{l}\text { Figueiredo SF, et al. } \\
\qquad(2013)\end{array}$ & $\begin{array}{l}\text { Hospital Amigo da } \\
\text { Criança: prevalência de } \\
\text { aleitamento materno } \\
\text { exclusivo aos seis meses e } \\
\text { fatores intervenientes }\end{array}$ & $\begin{array}{l}\text { Identificar o padrão de } \\
\text { AME nos primeiros seis } \\
\text { meses de vida de crianças } \\
\text { nascidas um Hospital } \\
\text { Amigo da Criança e os } \\
\text { fatores que contribuiram } \\
\text { para o desmame precoce. }\end{array}$ & $\begin{array}{l}\text { Pesquisa com uma coorte } \\
\text { prospectiva }\end{array}$ & $\begin{array}{c}\text { Os fatores que contribuiram } \\
\text { para o desmame precoce } \\
\text { foram: trabalho fora de casa, } \\
\text { mães que não } \\
\text { amamentaram } \\
\text { anteriormente e presença de } \\
\text { intercorrências mamárias. }\end{array}$ & $\begin{array}{c}\text { Revista Esc Enfermagem } \\
\text { USP }\end{array}$ \\
\hline Maciel VB, et al. (2013) & $\begin{array}{c}\text { Conhecimento de } \\
\text { gestantes e lactantes } \\
\text { sobre aleitamento materno } \\
\text { exclusivo }\end{array}$ & $\begin{array}{l}\text { Identificar o que as } \\
\text { gestantes e lactantes } \\
\text { afirmam sobre o que é o } \\
\text { aleitamento materno } \\
\text { exclusivo, qual a sua } \\
\text { duração e quais os } \\
\text { beneficios para a mãe e o } \\
\text { bebê. }\end{array}$ & $\begin{array}{l}\text { Estudo descritivo com } \\
\text { abordagem quantitativa }\end{array}$ & $\begin{array}{l}\text { Das entrevistadas, quanto a } \\
\text { definição de amamentação } \\
\text { exclusiva, } 25(55,6 \%) \\
\text { responderam que ela } \\
\text { consiste apenas no leite } \\
\text { materno; } 5(11,1 \%) \text { em leite } \\
\text { materno, água e chá; e } 12 \\
\text { (26,7\%) não conheciam o } \\
\text { significado do termo. Sobre } \\
\text { os beneficios, } 16(35,7 \%) \\
\text { afirmaram o aumento do } \\
\text { vínculo com o filho. }\end{array}$ & $\begin{array}{l}\text { Revista Brasileira em } \\
\text { Promoçăo da Saúde }\end{array}$ \\
\hline $\begin{array}{c}\text { Wander K e Mattison S, } \\
\text { (2013) }\end{array}$ & $\begin{array}{l}\text { The evolutionary ecology } \\
\text { of early weaning in } \\
\text { Kilimanjaro, Tanzania }\end{array}$ & $\begin{array}{c}\text { Avaliar a teoria do } \\
\text { investimento parenteral } \\
\text { em termos de risco para } \\
\text { desmame antes dos dois } \\
\text { anos de idade em } \\
\text { crianças de Kilimanjaro, } \\
\text { Tanzania. }\end{array}$ & Estudo longitudinal & $\begin{array}{l}\text { O peso foi um dos fatores } \\
\text { relacionados ao desmame } \\
\text { precoce. Crianças com } \\
\text { maior peso ao nascer } \\
\text { tiveram menor } \\
\text { probabilidade de } \\
\text { desmame precoce em } \\
\text { relação àquelas que } \\
\text { nasceram com baixo } \\
\text { peso. Não foram } \\
\text { observadas relações com } \\
\text { fonte de leite suplementar } \\
\text { (gado). }\end{array}$ & $\begin{array}{l}\text { Proceedings of the } \\
\text { Royal Society }\end{array}$ \\
\hline Oakley L, et al. (2014) & $\begin{array}{l}\text { The role of support and } \\
\text { other factors in early } \\
\text { breastfeeding cessation: } \\
\text { na analysis of data from } \\
\text { a maternity survey in } \\
\text { England }\end{array}$ & $\begin{array}{c}\text { Investigar a associação } \\
\text { entre o apoio pós-natal } \\
\text { e a interrupção da } \\
\text { amamentação aos } 10 \\
\text { dias e seis semanas no } \\
\text { bebê. }\end{array}$ & $\begin{array}{c}\text { Estudo retrospectivo } \\
\text { quantitativo }\end{array}$ & $\begin{array}{c}\text { Fatores } \\
\text { sociodemográficos } \\
\text { (idade materna, etnia, } \\
\text { país de nascimento, } \\
\text { privação, escolaridade) } \\
\text { e alimentação pré-natal, } \\
\text { foram associados a } \\
\text { interrupção da } \\
\text { amamentação aos } 10 \\
\text { dias e } 6 \text { semanas de } \\
\text { vida do bebê. }\end{array}$ & $\begin{array}{l}\text { BMC Pregnancy \& } \\
\text { Childbirth }\end{array}$ \\
\hline Rius JM, et al. (2014) & $\begin{array}{c}\text { Factores associados al } \\
\text { abandono precoz de la } \\
\text { lactância materna en } \\
\text { una región del este de } \\
\text { España }\end{array}$ & $\begin{array}{c}\text { Conhecer a prevalência } \\
\text { do Aleitamento Materno } \\
\text { durante os primeiros } 12 \\
\text { meses pós-parto e } \\
\text { analisar os fatores } \\
\text { associados ao seu } \\
\text { abandono }\end{array}$ & $\begin{array}{l}\text { Estudo prospectivo } \\
\text { quantitativo }\end{array}$ & $\begin{array}{l}\text { Os fatores associados } \\
\text { ao desmame precoce } \\
\text { foram: gravidez após } \\
\text { técnicas de reprodução } \\
\text { assistida, tabagismo } \\
\text { materno, expectativas } \\
\text { durante a lactação, uso } \\
\text { rotineiro de chupeta no } \\
\text { primeiro mês pós parto, } \\
\text { mãe com estudos } \\
\text { universitários e } \\
\text { sensação materna de } \\
\text { leite insuficiente. }\end{array}$ & Anales de Pediatría \\
\hline Roccil E, et al. (2014) & $\begin{array}{c}\text { Dificuldades no } \\
\text { aleitamento materno e } \\
\text { influência no desmame } \\
\text { precoce }\end{array}$ & $\begin{array}{l}\text { Verificar o tempo médio } \\
\text { do aleitamento materno } \\
\text { exclusivo (AME) de } \\
\text { crianças nascidas em } \\
\text { Hospital Amigo da } \\
\text { Criança (HAC) e } \\
\text { correlacionar o tempo } \\
\text { com as variáveis: } \\
\text { estado civil, idade } \\
\text { materna, peso do bebê, } \\
\text { dificuldades na } \\
\text { amamentação e } \\
\text { orientações recebidas. }\end{array}$ & Estudo de coorte & $\begin{array}{l}\text { Foram notadas } \\
\text { dificuldades no } \\
\text { aleitamento, como: } \\
\text { crença de leite fraco ou } \\
\text { pouco leite, volta ao } \\
\text { trabalho ou estudo, } \\
\text { trauma mamilar. }\end{array}$ & $\begin{array}{c}\text { Revista Brasileira de } \\
\text { Enfermagem }\end{array}$ \\
\hline Stuebe AM, et al. (2014) & $\begin{array}{l}\text { Prevalence and Risk } \\
\text { Factors for Early, } \\
\text { Undesired Weaning } \\
\text { Attributed to Lactation } \\
\text { Dysfuction }\end{array}$ & $\begin{array}{l}\text { Quantificar a prevalência } \\
\text { e identificar os fatores de } \\
\text { risco para o desmame } \\
\text { precoce e indesejado } \\
\text { que as mães atribuem às } \\
\text { dificuldades } \\
\text { imunológicas como a } \\
\text { amamentação. }\end{array}$ & Estudo longitudinal & $\begin{array}{c}\text { A prevalência de } \\
\text { lactação interrompida foi } \\
\text { de } 12 \text { por } 100 \text { mulheres } \\
\text { durante o primeiro ano } \\
\text { de vida do filho. Dentre } \\
\text { os fatores da chance de } \\
\text { ruptura da lactação, } \\
\text { considera-se mulheres } \\
\text { com sobrepeso ou } \\
\text { obesas, além de } \\
\text { sintomas depressivos } \\
\text { maternos. }\end{array}$ & $\begin{array}{c}\text { Journal of Women's } \\
\text { Health }\end{array}$ \\
\hline
\end{tabular}




\begin{tabular}{|c|c|c|c|c|c|}
\hline AUTORIANO & TÍTULO & OBJETIVO & $\begin{array}{l}\text { TIPO DE } \\
\text { ESTUDO }\end{array}$ & $\begin{array}{l}\text { PRINCIPAIS } \\
\text { RESULTADOS }\end{array}$ & $\begin{array}{c}\text { REVISTA/ } \\
\text { PUBLICAÇÃO }\end{array}$ \\
\hline $\begin{array}{l}\text { Figueiredo MC, et al. } \\
\qquad(2015)\end{array}$ & $\begin{array}{l}\text { Banco de Leite Humano: } \\
\text { apoio à amamentação e a } \\
\text { duração do aleitamento } \\
\text { materno exclusivo }\end{array}$ & $\begin{array}{l}\text { Identificar quais fatores } \\
\text { sociodemográficos estão } \\
\text { associados ao desmame } \\
\text { precoce e comparar a } \\
\text { duração do aleitamento } \\
\text { materno exclusivo entre } \\
\text { mães que receberam } \\
\text { orientações sobre } \\
\text { aleitamento materno e } \\
\text { mães que não receberam. }\end{array}$ & Estudo transversal & $\begin{array}{c}\text { Fatores socioeconômicos } \\
\text { como trabalho fora do lar, } \\
\text { menor número de filhos, } \\
\text { baixa escolaridade, } \\
\text { incentivo inadequado, } \\
\text { exercem influência negativa } \\
\text { na duração do aleitamento } \\
\text { materno exclusivo. Porém, a } \\
\text { não existência de cônjuges } \\
\text { acaba por reduzir a chance } \\
\text { da pausa da amamentação. }\end{array}$ & $\begin{array}{l}\text { Journal of Human Growth } \\
\text { and Development }\end{array}$ \\
\hline Oliveira CS, et al. (2015) & $\begin{array}{c}\text { Amamentação e as } \\
\text { intercorrếncias que } \\
\text { contribuem para o } \\
\text { desmame precoce }\end{array}$ & $\begin{array}{c}\text { Conhecer a vivência de } \\
\text { mães em réaçăo à } \\
\text { amamentação e as } \\
\text { intercorrências que } \\
\text { contribuem para o } \\
\text { desmame precoce. }\end{array}$ & $\begin{array}{l}\text { Estudo descritivo } \\
\text { exploratório }\end{array}$ & $\begin{array}{c}\text { Os principais fatores para o } \\
\text { desmame precoce foram: } \\
\text { déficit de conhecimento, } \\
\text { inexperiência/insegurança, } \\
\text { banalizaçấo das angústias } \\
\text { maternas, intercorrências da } \\
\text { mama puerperal, } \\
\text { interferências familiares, } \\
\text { leite fraco/insuficiente e } \\
\text { trabalho materno. }\end{array}$ & $\begin{array}{l}\text { Revista Gaúcha de } \\
\text { Enfermagem }\end{array}$ \\
\hline Teter MS, et al. (2015) & $\begin{array}{l}\text { Amamentação e } \\
\text { desmame precoce em } \\
\text { lactantes de Curitiba }\end{array}$ & $\begin{array}{l}\text { Identificar os fatores que } \\
\text { levam ao desmame } \\
\text { precoce em uma unidade } \\
\text { de saúde localizada no } \\
\text { município de Curitiba. }\end{array}$ & $\begin{array}{l}\text { Estudo descritivo } \\
\text { exploratório com análise } \\
\text { quantitativa das variáveis }\end{array}$ & $\begin{array}{c}\text { Sobre os motivos que } \\
\text { levaram ao desmame } \\
\text { precoce estavam: } 18,33 \% \\
\text { se devem ao pouco leite; } \\
18,33 \% \text { retorno ao } \\
\text { trabalho; } 10 \% \text { referiu que } \\
\text { o leite secou e } 6,67 \% \\
\text { referiram cansaço. }\end{array}$ & $\begin{array}{c}\text { Revista Espaço para a } \\
\text { Saúde }\end{array}$ \\
\hline Maciel VB, et al. (2016) & $\begin{array}{c}\text { Aleitamento Materno } \\
\text { em crianças indígenas } \\
\text { de dois municípios da } \\
\text { Amazônia Ocidental } \\
\text { Brasileira }\end{array}$ & $\begin{array}{l}\text { Analisar o aleitamento } \\
\text { materno de crianças } \\
\text { indígenas de zero a } \\
\text { dois anos e os fatores } \\
\text { associados ao } \\
\text { desmame. }\end{array}$ & $\begin{array}{l}\text { Estudo descritivo do } \\
\text { tipo transversal }\end{array}$ & $\begin{array}{c}\text { Estavam em } \\
\text { aleitamento materno } \\
60,6 \% \text { das crianças. Em } \\
\text { menores de } 6 \text { meses o } \\
\text { AME esteve presente } \\
\text { em } 35 \% \text { das crianças. A } \\
\text { única associação do } \\
\text { desmame precoce com } \\
\text { as variáveis foi a etnia. }\end{array}$ & $\begin{array}{l}\text { Acta Paulista de } \\
\text { Enfermagem }\end{array}$ \\
\hline Margotti E, et al. (2016) & $\begin{array}{c}\text { Fatores de risco para o } \\
\text { desmame precoce }\end{array}$ & $\begin{array}{c}\text { Determinar os fatores } \\
\text { de risco para o } \\
\text { desmame precoce. }\end{array}$ & Estudo de coorte & $\begin{array}{l}\text { Hospital não Amigo da } \\
\text { Criança, mãe que } \\
\text { trabalha fora do lar e } \\
\text { escore de Dimburgo se } \\
\text { mostraram como fatores } \\
\text { de risco para } \\
\text { amamentação exclusica } \\
\text { aos } 2 \text { e3 meses de vida } \\
\text { do bebê, e mulheres } \\
\text { com tendências } \\
\text { depressivas tiveram } \\
\text { predisposição ao } \\
\text { desmame. }\end{array}$ & Revista Rene \\
\hline Pang WW, et al. (2017) & $\begin{array}{l}\text { Direct vs Expresse } \\
\text { Breast Milk Feeding: } \\
\text { Relation to Duration of } \\
\text { Breastfeeding }\end{array}$ & $\begin{array}{l}\text { Analisar o aleitamento } \\
\text { materno direto e } \\
\text { expresso e sua } \\
\text { influência no tempo de } \\
\text { amamentação em uma } \\
\text { população asiática } \\
\text { multiétnica. }\end{array}$ & $\begin{array}{c}\text { Estudo retrospectivo } \\
\text { quantitativo }\end{array}$ & $\begin{array}{c}\text { Dentre os fatores } \\
\text { relacionados a uma } \\
\text { amamentação expressa } \\
\text { estavam: etnicidade } \\
\text { chinesa, educação } \\
\text { terciária, primiparidade } \\
\text { e emprego. A } \\
\text { amamentação expressa } \\
\text { teve a maior } \\
\text { probabilidade de } \\
\text { desmame precoce em } \\
\text { relação a amamentação } \\
\text { direta. }\end{array}$ & Nutrients \\
\hline Silva CS, et al. (2017) & $\begin{array}{l}\text { Association between } \\
\text { postpartum depression } \\
\text { and the practice of } \\
\text { exclusice breastfeeding } \\
\text { in the first }\end{array}$ & $\begin{array}{l}\text { Verificar a associação } \\
\text { entre a depressão pós- } \\
\text { parto e a ocorrência do } \\
\text { aleitamento materno } \\
\text { exclusivo. }\end{array}$ & $\begin{array}{l}\text { Estudo de coorte } \\
\text { transversal }\end{array}$ & $\begin{array}{c}\text { A amamentação } \\
\text { exclusica foi observada } \\
\text { em } 50,8 \% \text { das crianças e } \\
11,8 \% \text { das mulheres } \\
\text { apresentaram } \\
\text { sintomatologia indicativa } \\
\text { de depressão pós-parto. } \\
\text { Além disso o abandono } \\
\text { do AME também foi } \\
\text { relacionado a mulheres } \\
\text { mais jovens, que tinham } \\
\text { menor renda per capita e } \\
\text { que iniciaram seu pré- } \\
\text { natal tardiamente. }\end{array}$ & J Pediatr (Rio J) \\
\hline
\end{tabular}

Fonte: Dados da pesquisa, 2018. 


\section{DISCUSSÃO}

\section{Conhecimento sobre o aleitamento materno}

O leite materno é a melhor fonte de alimento para o lactente, pois possui a nutrição necessária que serve de proteção e promoção de saúde das crianças, além de possibilitar o crescimento e desenvolvimento das mesmas, devido ao alto poder nutricional e propriedades imunológicas (SALUSTIANO LP, et al., 2017).

Dessa forma, é importante, aproveitar tais benefícios com a prática do aleitamento materno exclusivo até os seis meses de vida do bebê, e somente depois, complementar o leite materno com outros tipos de alimentos até os dois anos de idade ou mais, como preconiza a Organização Mundial de Saúde (OMS) (WHO, 2016).

Assim, o conhecimento das mães sobre o aleitamento materno é fundamental segundo o estudo de Maciel VB, et al. (2013) e como a falta de informação sobre a importância do leite materno pode ser um fator para o desmame precoce. A pesquisa de Oliveira CS, et al. (2015) também confirma tal fato e aborda um ponto muito importante: as dúvidas maternas. Essas indagações podem ser por insegurança, inexperiência ou falta de conhecimento da mesma.

\section{Banalização das dúvidas maternas}

É importante salientar nesse aspecto que esses problemas de entendimento a respeito da amamentação podem ser por diversas causas, a exemplo do início do pré-natal tardio de acordo com Lima AP, et al., (2018), como também, "hospitais não amigo da criança" como diz Margotti E, et al. (2016), além da banalização por parte da equipe de saúde sobre as dúvidas maternas, muitas vezes devido a suposição errônea de que a mulher "saberá como fazer" (OLIVEIRA CS, et al., 2015).

Essa banalização por parte do profissional foi mostrada no estudo de Fialho FA, et al. (2014) como um dos principais pontos que levavam as mulheres a abandonar a amamentação. Dessa forma, pode-se compreender que a continuidade de ocorrência e geração de dúvidas, levarão as mães a suprir sua falta de conhecimento com ideias ou conhecimento empírico, o que pode levar muitas vezes a erros com relação a amamentação e assim ocasionar um desmame antes do tempo adequado.

\section{Crença do "leito fraco"}

Esse fato tem relação com os estudos de Oliveira CS, et al. (2015), Rius JM, et al. (2015), Roccil E, et al. (2014) e Teter MS, et al. (2015) que afirmam que um dos mitos extremamente comum é a crença materna de produzir uma menor quantidade de leite, "leite fraco", ou que o mesmo não é suficiente para nutrir o bebê. Essa relação é pontuada por Oliveira CS, et al. (2015) por algumas questões. De início pela ideia de que o leite da mãe é muito ralo, ou desconhecer aspectos fisiológicos do próprio organismo o que leva a mãe a pensar que esse alimento não é suficiente para sua prole. Além disso, outro aspecto importante e que muitas vezes reforça a ideia de "leite fraco" é o choro infantil constante, que leva a mulher a associar tal comportamento com fome, entretanto, nem sempre é verdade. Esse fato entra em concordância com o estudo de revisão bibliográfica publicado por Fialho FA, et al. (2014), que observou que esse entendimento sobre o choro infantil e leite fraco é uma das principais causas para o desmame.

Também concorda com a ideia da "falta do leite" a publicação com o título "A prática do aleitamento materno e os fatores que levam ao desmame precoce: uma revisão integrativa" de Lima AP, et al. (2018) na qual afirma que esse fator é um ponto que gera o desmame, pois, essa crença errônea, além de ser citado em vários dos artigos por eles pesquisados, leva a nutriz a ter insegurança e acrescentar outros alimentos na dieta infantil antes do tempo, como chás, água e outros líquidos.

\section{Influência familiar}

Assim, é interessante o destaque de que muitas vezes essa decisão de acrescentar outros alimentos na dieta é tomada por meio da influência familiar, o que se percebe nas pesquisas da presente revisão sistemática, principalmente no que diz respeito a avó materna. A figura da avó possui a herança da sua própria 
experiência como mãe e tem, na maioria das vezes, como base o senso comum o que acaba por convencer a nutriz de adicionar outros produtos na alimentação (OLIVEIRA CS, et al., 2015).

Esse tema é abordado também no Caderno de Atenção Básica, Saúde da criança: aleitamento materno e alimentação complementar, e afirma que a prática da amamentação é fortemente influenciada pelo meio em que o binômio mãe-filho se encontra, assim, a opinião das avós é de grande importância para a mulher. Entretanto, segundo o estudo de Deus MD e Dias AC (2016), o conhecimento das avós pode ter um lado negativo, em relação a falta de estimulação da prática do aleitamento materno exclusivo, pela inserção de outros alimentos complementares na dieta do bebê.

\section{Trauma mamilar}

Entre outros fatores para o desmame precoce, tem-se o trauma mamilar (OLIVEIRA CS, et. al, 2015; ROCCIL E, et al., 2014; FIGUEIREDO SF, et al., 2013). Tais estudos concordam com a revisão realizada por Lima AP, et al. (2018) onde se aponta que os machucados na mama são questões de risco para a pausa na amamentação pela extrema dor que a mãe sente na hora da alimentação do infante. É importante salientar nesse ponto como tais situações podem ser evitadas, já que, através de uma orientação adequada a respeito da pega correta da criança no momento da amamentação esses ferimentos poderiam ser reduzidos, entretanto, infelizmente essa orientação muitas vezes não é contemplada pelos fatos já citados aqui (OLIVEIRA CS et al., 2015).

\section{Uso de chupetas}

O uso de chupetas também é considerado como fator de risco para desmamar precocemente de acordo com Rius JM, et al. (2015), o que corrobora com o artigo de revisão publicado por Lima AP, et al. (2018), no qual aponta, que apesar dos bicos artificiais sirvam de acalento para o bebê, nos primeiros dias, eles podem gerar uma menor quantidade de mamadas por parte da criança, e como nesse período inicial o organismo feminino está se acostumando com a quantidade de leite necessárias a ser produzida, essa situação pode gerar uma diminuição de vezes que ocorre a sucção o que leva a uma menor quantidade de leite produzido devido as frequências reduzidas da amamentação.

\section{Retorno da mãe ao trabalho}

O retorno as atividades laborais ou aos estudos é outro fator extremamente citado pelos autores Figueiredo SF, et al. (2013), Figueiredo MC, et al. (2015), Margotti E, et al. (2016), Oliveira CS, et al. (2015), Rius JM, et al. (2014), Roccil E, et al. (2014), Teter MS, et al. (2015), que concordam com o artigo de revisão publicado por Lima AP, et al. (2018) onde aponta que a independência da mulher faz com que exista a necessidade, por parte dela, de voltar ao mercado de trabalho o que é fator para que não haja estimulação adequada dos seios diminuindo a quantidade de leite materno.

\section{Depressão}

A depressão pós-parto também é um quesito muito relatado dentre os artigos encontrados como o de Margotti E, et. al (2016), Silva CS et al. (2017) e Stuebe AM, et al. (2014). Dessa forma é importante a investigação da saúde mental da mãe pela equipe que a acolhe tanto no pré-natal quanto no pós-natal.

\section{Equipe de saúde no pré-natal}

As recomendações por meio de profissionais da saúde também são um ponto preocupante em relação ao desmame precoce que, às vezes, pode ser gerado por falta de capacitação de pediatras no manejo da amamentação, pois algumas vezes indicam leite em formas suplementares (ROCCIL E, et al., 2014).

Esse dado concorda com o exposto em uma revisão realizada por Fialho FA, et al. (2014), que sugere que algumas recomendações realizadas por pediatras nos primeiros meses de vida, por exemplo, introduzir alguns alimentos de maneira desnecessária, interferem na amamentação exclusiva do bebê.

Esse fato pode gerar uma menor sucção da criança ao seio materno e diminuir a quantidade de leite produzida como já exposto anteriormente. 


\section{Presença do parceiro da mãe}

A presença do parceiro da mãe foi algo extremamente interessante observado na pesquisa, pois de acordo com Figueiredo MC, et al. (2015) a não existência de um companheiro reduzia a chance da mulher de abandonar a amamentação.

O artigo publicado por Fialho FA, et al. (2014) traz questões importantes para a discussão desse ponto, pois de acordo com ele, mesmo que existam parceiros que estimulam o processo de amamentação, outros, por aspectos de ciúmes, disfunção sexual, ansiedade e rejeição, podem levar a mãe a querer abandonar a prática, assim, a equipe de saúde deve ficar atenta a esse aspecto e não só trabalhar com a conscientização da mãe, mas também do seu núcleo familiar.

\section{Etnia}

O fator "etnia" também foi considerado nos estudos da revisão, com base nas publicações de Maciel VB, et al. (2016), que trata da relação da amamentação em povos indígenas e Pang WW, et al. (2017), que estuda, a prevalência de aleitamento materno e direto em uma cidade na China.

Nos dois estudos, a etnia era considerada como um fator para o desmame precoce, com base em análises quantitativas. Poucos estudos fazem relação com a amamentação e etnias, e não se sabe ao certo a influência da praticas da amamentação sobre a etnicidade.

\section{Peso da criança}

A questão do peso da criança também é um fator relevante. Segundo os estudos de Wander $\mathrm{K}$ e Mattison S, (2013), quanto menor o peso do bebê ao nascer, maior a probabilidade do desmame precoce.

É visto, dessa forma, que as baixas taxas de Aleitamento materno em bebês prematuros se devem pela dificuldade em amamentá-los, devido a fatores como coordenação sucção-deglutição-respiração, hospitalização prolongada e estresse materno em decorrência do sentimento de incapacidade em amamentar o filho.

\section{Fatores sociodemográficos}

Por último, é importante salientar os aspectos socioeconômicos no qual as mulheres se encontram, pois, eles influenciam de maneira direta na amamentação infantil.

De acordo com os artigos selecionados podemos destacar: mulheres mais jovens, citado por Silva CS, et al. (2017), menor renda per capita também citado por Silva CS, et al. (2017) e Figueiredo MC, et al. (2015), escolaridade mais baixa e menor quantidade de filhos (FIGUEIREDO MC, 2015).

Haja vista, uma suposição plausível é que tais grupos se encontram em uma situação com menor informação que explica o porquê de acabarem deixando de amamentar corretamente, afinal, a falta de conhecimento é um ponto crucial para a realização desmame precoce, como já citado, concordando com o encontrado por Fialho AP, et al. (2014), que observou que aquelas com menor grau de escolaridade ou que são primíparas tem menos conhecimento e retiram mais cedo a amamentação da criança.

\section{CONCLUSÃO}

Ao final dessa revisão foi possível observar quais os principais fatores que influenciam no desmame precoce sendo eles: acreditar que possuí leite fraco ou insuficiente; trauma mamilar; voltar ao trabalho ou estudos; interferências externas sejam elas por conta de algum profissional da área da saúde ou familiar; depressão pós-parto; questões socioeconômicas; etnia e baixo peso da criança.

Esses dados são de suma importância para a comunidade cientifica tendo em vista a importância da amamentação da criança para a saúde e bem-estar populacional. Dessa forma, pode-se sugerir a realização de políticas públicas que reduzam o desmame precoce ou rastreamento precoce no pré-natal das mulheres que possuem esse risco. 


\section{REFERÊNCIAS}

1. BRASIL. Diretrizes Metodológicas: elaboração de revisão sistemática de estudos observacionais comparativos sobre fatores de risco e prognóstico/Ministério da Saúde. Secretaria de Ciência, Tecnologia e Insumos Estratégicos. Departamento de Ciência e Tecnologia - Brasília: Ministério da Saúde, 2014.

2. BRASIL, Ministério da Saúde Secretaria de Atenção à Saúde. Departamento de Atenção Básica. Saúde da criança: aleitamento materno e alimentação complementar Brasília: Ministério da Saúde; 2 ed., 2015.

3. DEUS MD, DIAS ACG. Avós cuidadores e suas funções: uma revisão integrativa da literatura. Pensando Fam, n.1, v. 20, p.112-25, 2016.

4. FIALHO F, et al. Fatores associados ao desmame precoce do aleitamento materno. Rev Cuid. n.1, v.5, p. 670-8, 2014.

5. FIGUEIREDO MCD, BUENO MP, RIBEIRO CC, et al. Banco de Leite Humano: O Apoio à Amamentação e a Duração do Aleitamento Materno Exclusivo. Journal of Human Growth and Development. n. 2, v. 25. p.204-210, 2015.

6. FIGUEIREDO SF, MATTAR MJG, ABRÃO ACFV. Hospital Amigo da Criança: prevalência de aleitamento materno exclusivo aos seis meses e fatores intervenientes. Rev Esc Enferm USP. N. 6, v. 47, p. 1291-7, 2013.

7. LIMA APC, NASCIMENTO DS, MARTINS MMF. A prática do aleitamento materno e os fatores que levam ao desmame precoce: uma revisão intergrativa. Journal of Health Biol Sci, 2018.

8. MACIEL VBS, SILVA RP, SANUDO A, et al. Aleitamento materno em crianças indígenas de dois municípios da Amazônia Ocidental Brasileira. Acta Paul Enferm. n.4, v.29, p. 469-75, 2016.

9. MARGOTTI E, MATTIELO R. Fatores de risco para o desmame precoce. Rev Rene. n. 4, v. 17, p. 537-44, 2016.

10. MONTRONE VG, ARANTES CIS. Prevalência do aleitamento materno na cidade de São Carlos, São Paulo. J Pediatr, 2000.

11. OAKLEY LL, HENDERSON J, REDSHOW M, et al. The role of support and other factors in early breastfeeding cessation: an analysis of data from maternity survey in England. BMC Medicine, article number 88, 2014.

12. OLIVEIRA CS, IOCCA FA, CARRIJO MLR, et al. Amamentação e as intercorrências que contribuem para o desmame precoce. Rev Gaúcha Enferm. v. 36, p.16-23, 2015.

13. OPAS/OMS. Amamantar: amor y salud para siempre. Disponível em: https://www.paho.org/hq/index.php?option=com_content\&view=article\&id=9305\&ltemid=42397\&lang=es. Acessado em: 11/05/2018.

14. OPAS/OMS BRASIL. Amamentação: conheça as novas orientações da OMS e UNICEF. Disponível em: http://www.paho.org/bra/index.php?option=com_content\&view=article\&id=5631:oms-e-unicef-lancam-novasorientacoes-para-promover-aleitamento-materno-em-unidades-de-saude-de-todo-o-mundo\&ltemid=820. Acessado em: $11 / 05 / 2018$.

15. PANG WWP et al. Direct vs. Expressed Breast Milk Feeding: Relation to Duration of Breastfeeding. Nutrients, n.9, V.547, 2017.

16. RIUS JM, ORTUNÕ J, RIVAS C, et al. Factores associados al abandono precoz de la lactancia materna en uma región del este de España. Asociacíon de Pediatría. Elsevier Espanha, 2014.

17. ROCCIL E, FERNANDES RAQ. Difi culdades no aleitamento materno e influencia no desmame precoce. Rev Bras Enferm. n. 1, v. 67, p. 22-7, 2014.

18. SALUSTIANO LPQ, ABADALLAH VOS, PINTO RMC. Fatores associados à duração do aleitamento materno em crianças menores de seis meses. Rev. Bras. Ginecol. Obstet, n. 1, v. 34, p. 28-33, 2012.

19. SILVA CS, LIMA MC, SEQUEIRA ALA, et al. Association between postpartum depression and the practice of exclusive breastfeeding in the first three months of life. J Pediatr (Rio J). ;v. 93, p.356-64, 2017.

20. STUEBE AM, HORTON BJ, CHETWYND E, et al. Prevalence and Risk Factors for Early, Undesired Weaning Attributed to Lactation Dysfunction. Journal of Women's Health, Volume 23, Number 5, 2014.

21. TETER MSH, OSELAME GB, NEVES EB. Amamentação e Desmame Precoce em Lactantes de Curitiba. Rev Espaço para a Saúde, v.16, n.4. Londrina, 2015.

22. UNICEF BRASIL. Aleitamento Materno. Disponível em: https://www.unicef.org/brazil/pt/activities_10003.htm. Acessado em: 11/05/2018.

23. VICTORA CG, BARROS AJ, FRANÇA GV, et al. Breastfeeding in the 21st century: epidemiology, mechanisms, and lifelong effect. Lancet. 2016.

24. WANDER K, MATTISON S. The Evolutionary ecology of early weaning in Kilimanjaro, Tanzania. Proceedings of the Royal Society B 280, 2013.

25. WHO. Guideline: protecting, promoting and supporting breastfeeding in facilities providing maternity and newborn services. Geneva, 2017.

26. WHO, World Health Organization. Maternal, newborn, child and adolescent health. Breastfeeding, 2016. 